\title{
Functional properties of accessory AV pathways during premature atrial stimulation
}

\author{
Agustin Castellanos, Jr., Cesar A. Castillo, Abdul S. Agha, B. Befeler, and Robert J. Myerburg \\ From the Cardiovascular Laboratory, Veterans Administration Hospital, and the Division of Cardiology, \\ Department of Medicine, University of Miami School of Medicine, Miami, Florida, U.S.A.
}

Premature atrial stimulation was performed during His bundle recording in four patients with the type of ventricular pre-excitation in which the forward $H$ deflection appeared after the onset of the delta wave. The $A V$ conduction patterns occurring in these patients seemed to have depended on the physiological differences existing between normal pathway and accessory pathway $(A P)$ at different moments of the cycle. These differences explained the patterns noted in driven beats, namely, exclusively accessory pathway conduction (Case I), combined accessory and normal pathway conduction (Case 2), and exclusive normal pathway conduction (Cases 3 and 4).

In Cases $I$ and 3 the effective refractory period of the AP was longer than that of the normal pathway. Moreover, Case 3 had AP conduction only towards mid-cycle, sandwiched in between periods of normal pathway conduction. In Case 2, with very short refractory periods (less than 170 msec), premature atrial impulses reached the ventricles during the peak of the $T$ wave. Case 4 had normal pathway conduction with right bundle-branch block in sinus, driven, and late diastolic testing beats, with AP conduction only at the shorter coupling intervals. The ventricular rates of spontaneous or induced atrial tachyarrhythmias were significantly faster when the accessory pathway's effective refractory period was short (Cases 2 and 4), than in the other two (Cases I and 3) with longer effective refractory periods. Though premature atrial stimulation has increased our knowledge of $A V$ conduction, more studies are necessary to extend their usefulness to other patients with pre-excitation syndrome.

Clinical and experimental studies have shown the importance of premature atrial stimulation in analysing the physiological properties of the AV node and ventricular specialized conducting system (Moe, Mendez, and Han, 1965; Damato et al., 1969; Wit et al., 1970a, b; Wellens, 1971 ; Touboul et al., I972). It, therefore, appeared that intermittent paired atrial pacing could also provide additional information regarding the $\mathrm{AV}$ conduction patterns seen in patients with pre-excitation (WPW) syndrome.

\section{Subjects and methods}

The technique of $\mathrm{His}$ bundle recordings used in our department has been described previously (Castellanos et al., 1970; Castillo and Castellanos, 1970; Castellanos and Castillo, 1972). Several intracardiac leads were recorded simultaneously with four surface leads. The stimulator used delivered slightly underdamped pulses, $2.5 \mathrm{msec}$ in duration. The right atrium was paced at the

Received I4 September 1972. lowest rate that persistently suppressed sinus node activity. These driving stimuli $\left(\mathrm{St}_{1}\right)$ had twice diastolic threshold intensity. By means of a variable refractory delay, testing stimuli $\left(\mathrm{St}_{2}\right)$ were delivered after every eighth driven beat at 10 to $15 \mathrm{msec}$ intervals to scan the cycle. The intensity of $\mathrm{St}_{2}$ was twice that of $\mathrm{St}_{1}$. Four patients with the type of pre-excitation in which the forward $\mathbf{H}$ deflection was inscribed after the onset of ventricular depolarization were studied (Castellanos et al., 1970; Castillo and Castellanos, 1970; Massumi and Vera, I97I; Coumel et al., 1971). Spontaneous and induced tachyarrhythmias occurred in all patients.

\section{Definition of terms}

The A-H (low right atrium to $\mathrm{His}$ interval) as measured in the His bundle lead was taken as a measure of $\mathrm{AV}$ node conduction time (normal values: 50 to $120 \mathrm{msec}$ ).

In beats with exclusive conduction through the normal pathway the $\mathrm{H}-\mathrm{V}$ (His to-ventricle) interval was taken as a measure of His-Purkinje conduction time (normal value: 35 to $55 \mathrm{msec}$ ). 
In beats with any degree of pre-excitation, the A-H interval could still be used to determine AV node conduction time. However, the H-V (or V-H) interval only represented differences in activation time of His bundle (by the impulse traversing the AV node) and ventricles (by the impulse descending through the accessory pathway). In all beats ' $V$ ' indicated the onset of ventricular depolarization in whichever lead it occurred first, irrespective of the pathway (s) that the impulse used to activate the ventricles.

$A_{1}, H_{1}$, and $V_{1}$ as well as $A_{2}, H_{2}$, and $V_{1}$ were used in reference to the corresponding responses at the low right atrium, His bundle, and ventricles, induced by driving and testing stimuli, respectively.

The effective refractory period (ERP) of the normal pathway was the longest $A_{1}-A_{2}$ interval at which $A_{2}$ failed to activate the ventricles through the normal pathway. The effective refractory period of the accessory pathway was the longest $A_{1}-A_{2}$ interval at which $A_{2}$ failed to produce a $Q R S$ complex with a delta wave.

\section{Case reports}

Case I The recordings shown in Fig. I and 2 were obtained from a 63-year-old man with Wolff-ParkinsonWhite (WPW) type B. Though various types of atrial tachyarrhythmias were observed in this patient (including atrial fibrillation), ventricular rates did not exceed I90 a minute. During sinus rhythm (Fig. I, left) the PR interval and QRS complexes measured $120 \mathrm{msec}$ and $130 \mathrm{msec}$, respectively. The forward $\mathrm{H}$ deflection coincided with the beginning of ventricular $(\mathrm{V})$ depolarization. Hence, the QRS complex was interpreted as a 'combination', or 'fusion' beat in which the ventricles were activated through both, normal and accessory, AV communications. Spontaneous atrial extrasystoles occurring in mid-diastole (Fig. I, right) propagated to the ventricles predominantly, or exclusively, through the accessory pathway. Hence, the $\mathrm{H}$ deflection was lost within the ventricular electrogram recorded by the $\mathrm{His}$ bundle electrogram lead. The PR interval and QRS complexes measured 120 and $140 \mathrm{msec}$, respectively.

Driving stimuli, and testing stimuli delivered at coupling intervals ranging between 600 and $350 \mathrm{msec}$, reached the ventricles exclusively through the accessory pathway (Fig. 2, left-sided panel). However, when the coupling interval was reduced to $340 \mathrm{msec}$, the effective refractory period of the accessory pathway was reached. Hence, the atrial impulse propagated exclusively through the normal pathway (with increasing degrees of $\mathrm{AV}$ nodal delay) until the effective refractory period of the latter occurred at a $A_{1}-A_{2}$ interval of $265 \mathrm{msec}$. During this early part of the cycle (last beat in the right-sided panel of Fig. 2) the $\mathrm{St}_{2}-\mathrm{V}_{2}$ intervals became longer than the $\mathrm{St}_{1}-\mathrm{V}_{1}$ intervals. Moreover, an $\mathrm{H}$ deflection now appeared $45 \mathrm{msec}$ before the onset of a ventricular complex showing the characteristics of left anterior hemiblock. QRS duration was $90 \mathrm{msec}$.

To summarize, in this patient the effective refractory period of the accessory pathway was longer than that of the normal AV pathway.

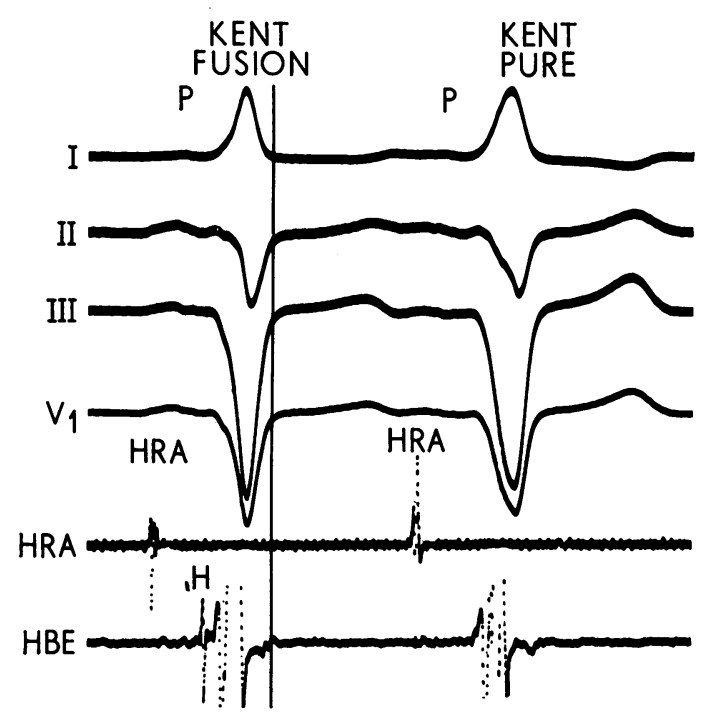

FIG. I Case I. His bundle recordings in a patient with Wolff-Parkinson-White type $B$. The first $Q R S$ complex was a 'combination' or 'fusion' beat. The spontaneous premature atrial beat (right) was conducted to the ventricles exclusively through the accessory pathway. HRA, high right atrium; HBE, His bundle electrogram. In this and all the illustrations, paper speed was $100 \mathrm{~mm} / \mathrm{sec}$.

Case 2 The tracings presented in Fig. 3 and 4 were obtained from a 27-year-old patient with WPW type A who had frequent bouts of atrial flutter and/or fibrillation with ventricular rates as high as 3 IO per minute.

The $S_{1}-V_{1}$ intervals and the QRS complexes had a duration of 130 and $135 \mathrm{msec}$, respectively. Inscription of the forward $\mathrm{H}$ deflection slightly after the onset of depolarization suggested that ventricular depolarization occurred through both normal and accessory pathways (first two QRS complexes in Fig. 3). As the coupling intervals were reduced, the expected AV nodal delay occurred. Therefore, the forward $\mathbf{H}$ deflections were gradually lost within the ventricular electrogram of the His bundle electrogram lead until exclusive AP conduction appeared (last beat in Fig. 3). In contrast to what occurred in Case I, Fig. 4 shows that atrial impulses could still traverse the AP at coupling intervals as short as $170 \mathrm{msec}$. The resulting (last) ventricular complex appeared at the peak of the antecedent $T$ wave. The increase in $\mathrm{QRS}$ duration and distortion probably reflected the slow, irregular, conduction occurring during the period of incomplete ventricular recovery.

To summarize, in this patient the effective refractory period of the accessory pathway appeared to have been extremely short.

Case 3 Fig. 5 and 6 were obtained from a 28-year-old man with intermittent WPW type A. The arrhythmias 


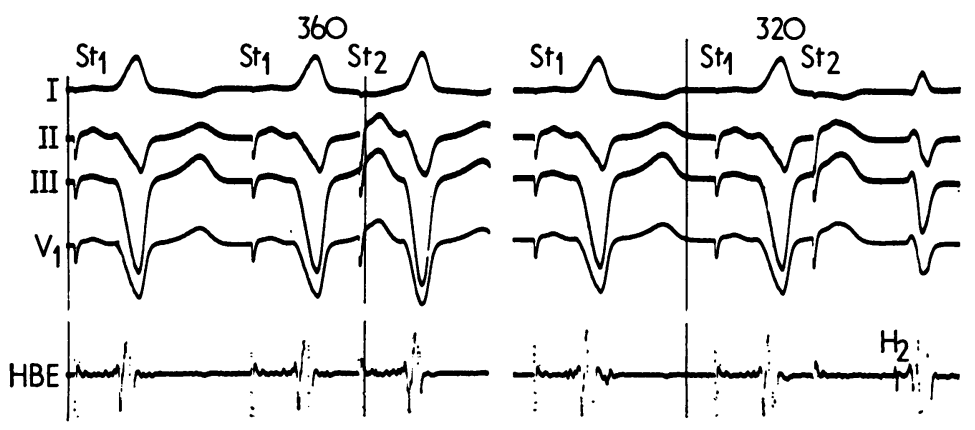

FIG. 2 Case I. Premature atrial stimulation in a patient showing exclusive accessory pathway conduction in driven $\left(S t_{1}\right)$ and late diastolic testing $\left(S t_{2}\right)$ beats (left-sided panel). Exclusive normal pathway conduction occurred at shorter coupling intervals (right-sided panel). Driving cycle length was $600 \mathrm{msec}$.

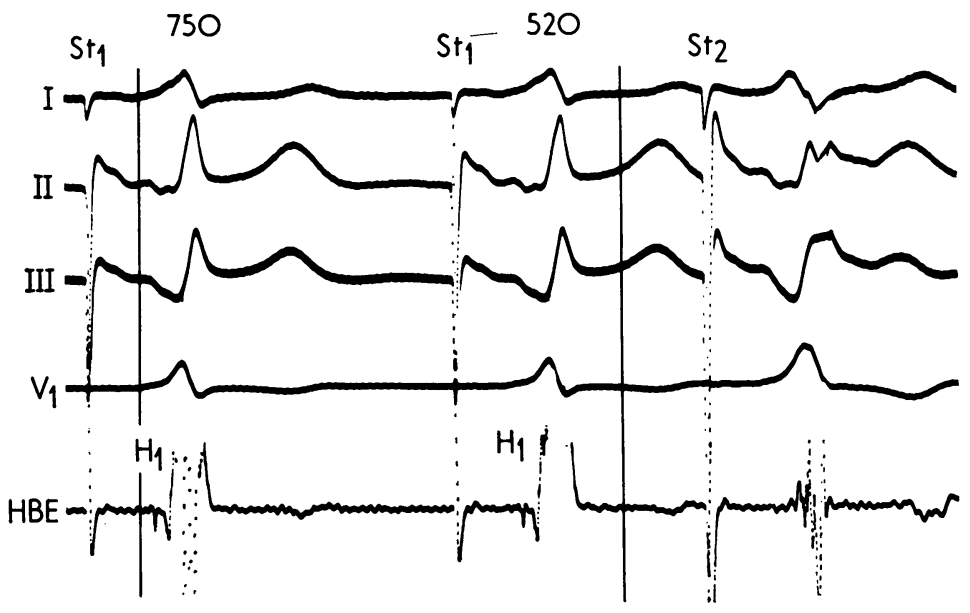

FI . 3 Case 2. Premature atrial stimulation in a patient with Wolff-Parkinson-White type A. The first two (driven) QRS complexes were 'combination' beats in which the forward His bundle deflection $\left(H_{1}\right)$ was inscribed after the beginning of the delta wave. The premature atrial stimulus $\left(S t_{2}\right)$ was conducted to the ventricles predominantly through the accessory pathway. Hence, the forward $H$ deflection was lost within the electrogram recorded by the His bundle electrogram lead. Driving cycle length was $750 \mathrm{msec}$.

recorded in this patient were only of supraventricular contour in which the rate did not exceed $180 \times \mathrm{min}$. It was interesting to note that sinus and driven impulses, as well as coupled stimuli delivered at coupling intervals ranging between 680 and $600 \mathrm{msec}$, reached the ventricles exclusively through the normal AV pathway. The QRS complexes were of normal range (first two beats in Fig. 5); namely: $\mathrm{St}_{1}-\mathrm{V}_{1}=175 \mathrm{msec}, \mathrm{A}_{1}-\mathrm{H}_{1}=85$ msec, and $\mathrm{H}_{1}-\mathrm{V}_{1}=40 \mathrm{msec}$.

However, at $A_{1}-A_{2}$ intervals between 560 and 420 msec the QRS morphology became that of WPW type A (last beat in Fig. 5).

The $\mathrm{St}_{2}-\mathrm{V}_{2}$ interval was shorter than the corresponding driven interval. Inscription of the forward $\mathrm{H}$ deflec- tion after the onset of the delta wave suggested ventricular pre-excitation through an extranodal, extra-Hisian communication. At coupling intervals between $4 \mathrm{IO}$ and $315 \mathrm{msec}$ the ventricular complexes again became of normal configuration. The forward $\mathbf{H}$ deflection appeared $40 \mathrm{msec}$ before the onset of ventricular depolarization. In addition, during this (early) part of the cycle the $\mathrm{St}_{2}-\mathrm{V}_{2}$ interval was prolonged at the expense of the $\mathrm{AH}$ interval due to expected (relative) AV nodal refractoriness (last beat in Fig. 6). Finally, when the $A_{1}-A_{2}$ interval was reduced to $310 \mathrm{msec}, A_{2}$ failed to reach the His bundle and consequently, the ventricles.

To summarize, AP conduction only occurred towards the middle of the cycle, in between periods of exclusive normal pathway conduction with normal AV intervals 


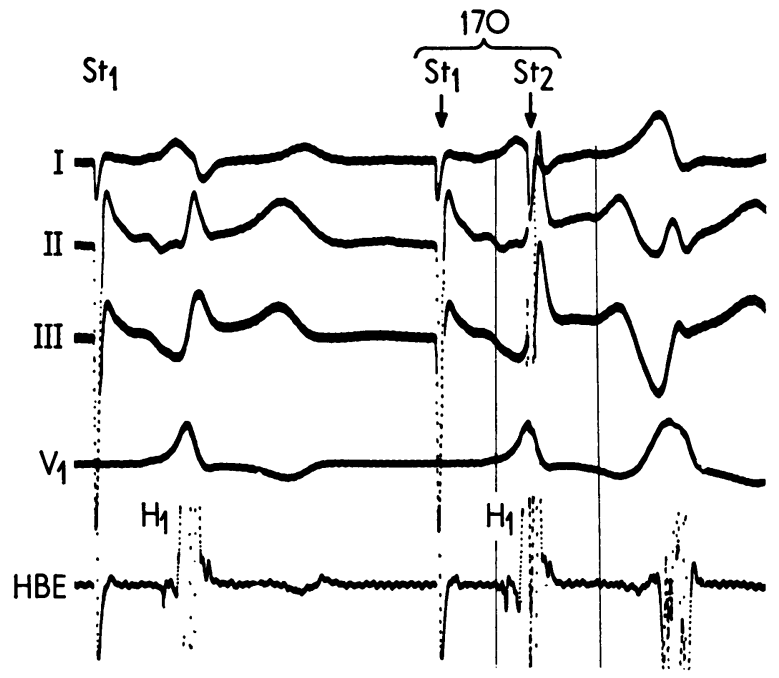

FIG. 4 Case 2. Premature atrial stimulation in a patient with Wolff-Parkinson-White type A. Note that $A V$ conduction could still occur through the accessory pathway at a very short coupling interval (170 msec). Hence, the premature (last) QRS complex appeared on top of the antecedent $T$ wave.

in late diastole, and prolonged $\mathrm{AV}$ intervals (earlier in the cycle).

Case 4 Fig. 7 was obtained from a 52-year-old man with WPW type A and a long-standing history of palpitations who developed right bundle-branch block in 1970. The arrhythmias noted in this patient were of several types, namely reciprocating tachycardia with exclusive His bundle conduction, and bouts of rapid atrial fibrillation or atrial flutter with bizarre QRS complexes. Exclusive conduction through the normal pathway (with right bundle-branch block) occurred in sinus, driven, and late diastolic testing beats (first two QRS complexes in Fig. 7). The duration of the corre-

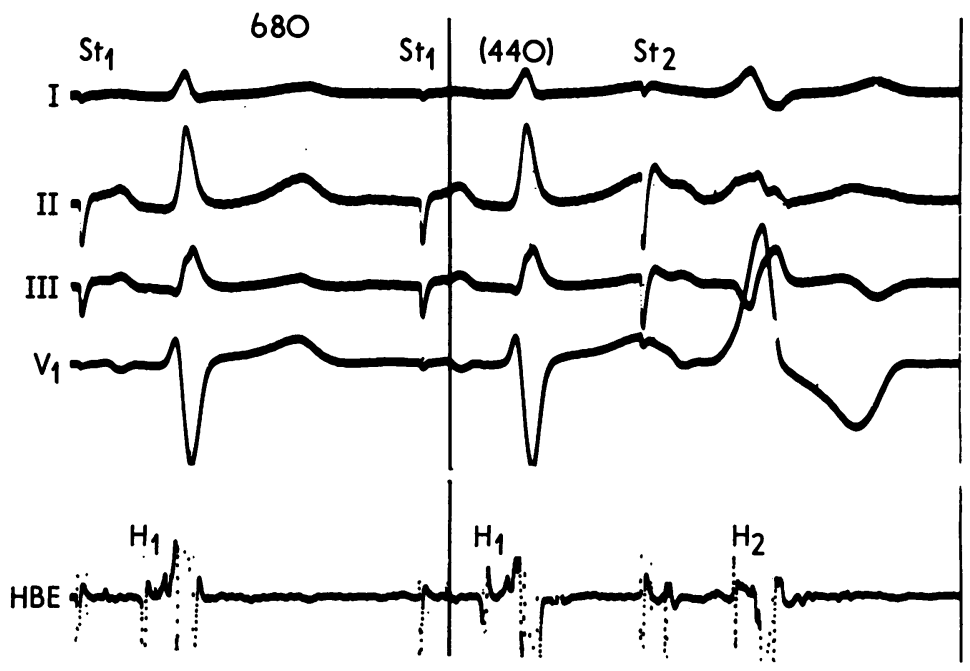

FIG. 5 Case 3. His bundle recordings in a patient with intermittent Wolff-Parkinson-White type A. The driven beats show exclusive normal pathway conduction. The premature (last) atrial impulse was conducted through the accessory pathway. Hence, the St-V interval became shorter and the forward $H$ deflection was inscribed after the onset of the delta wave. 

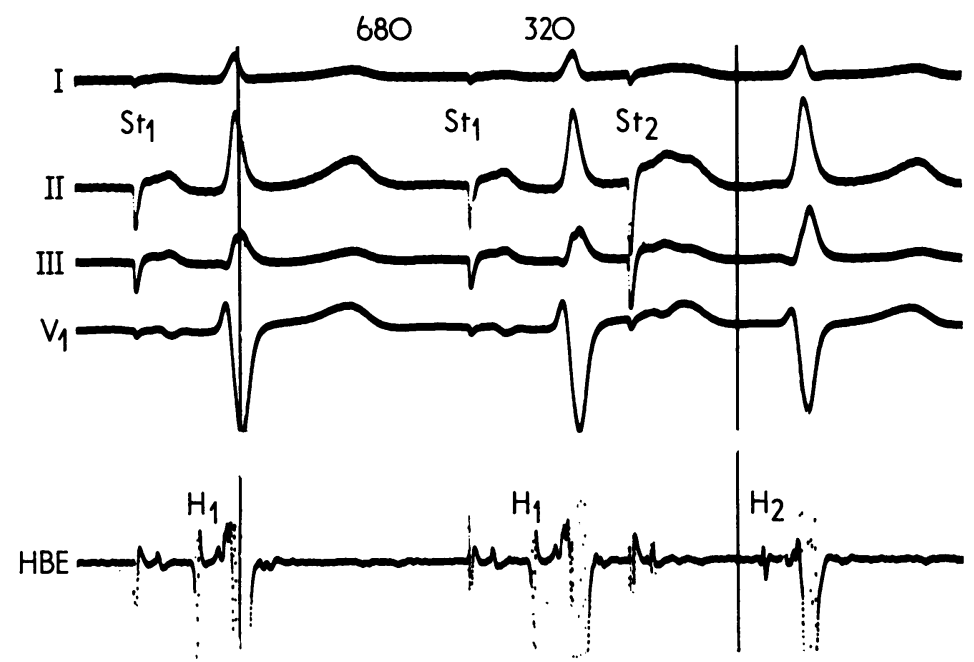

FIG. 6 Case 3. His bundle recordings showing exclusive normal pathway conduction with normal $S t-V$ intervals in driven beats, and with prolonged St-V intervals at a coupling of 320 msec.

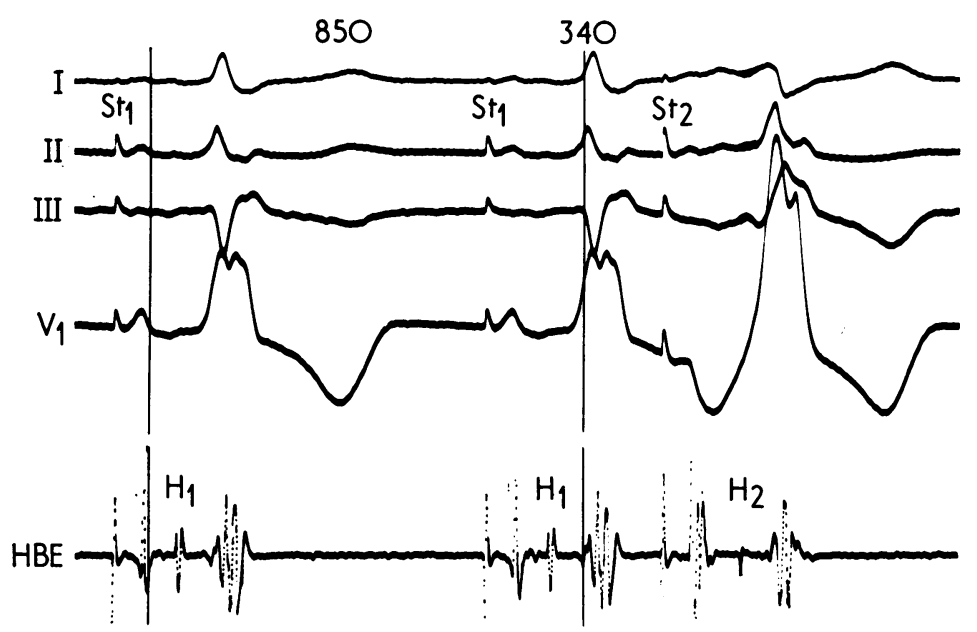

FIG. 7 Case 4. His bundle recordings in a patient with intermittent Wolff-Parkinson-White type $A$. Driven beats $\left(S t_{1}\right)$ showed exclusive normal pathway conduction with 'complete' block in the right bundle-branch. The $H$ deflection appeared $55 \mathrm{msec}$ before the onset of ventricular depolarization. On the contrary the premature (last) atrial stimulus was conducted through the accessory pathway with type $A$ morphology. The latter was not due to right bundle-branch block since the forward $H$ deflection was inscribed after the beginning of the QRS complex. Driving cycle length was $850 \mathrm{msec}$. 
sponding intervals was as follows: $A_{1}-H_{1}=85 \mathrm{msec}$, $\mathrm{H}_{1}-\mathrm{V}_{1}=55 \mathrm{msec}$, and $\mathrm{St}_{1}-\mathrm{V}_{1}=\mathrm{r} 80 \mathrm{msec}$. A similar morphology (indicative of normal pathway conduction with right bundle-branch block) was also observed at coupling intervals between 850 and 570 . When the $A_{1}-A_{2}$ intervals ranged between 560 and 290 the forward $\mathrm{H}$ deflection was inscribed after (or close to) the onset of the QRS complex. Hence, the $\mathrm{St}_{2}-\mathrm{V}_{2}$ interval became shorter than the corresponding driving interval. This indicated that the ventricles were pre-excited ahead of the His-bundle. During this part of the cycle the QRS morphology was that of WPW type $A$ as shown by the last beat of Fig. 7 .

Testing stimuli were not delivered at coupling intervals of less than $290 \mathrm{msec}$ because of the prolonged bouts of tachyarrhythmias which were constantly elicited. Though the exact duration of the effective refractory period of the accessory pathway could not be determined with certainty its value was less than $290 \mathrm{msec}$.

\section{Discussion}

Previous studies have emphasized the usefulness of His bundle recordings, combined with atrial pacing at increasing rates, in enhancing our knowledge of the pre-excitation syndrome (Castellanos et al., 1970; Castillo and Castellanos, 1970; Wellens, 197I ; Massumi and Vera, 197I; Coumel et al., 1971; Touboul et al., 1972).

This report shows that premature atrial stimulation complements the latter technique. Moreover, it offers additional information regarding the complex AV conduction patterns seen in patients in whom the forward $\mathrm{H}$ deflection appeared (at one moment or another) after the onset of ventricular depolarization.

The various responses to coupled atrial pacing most probably reflected the physiological differences existing between normal and accessory pathways in different moments of the cycle. This statement also applies to the AV conduction patterns observed after driven stimuli, which consisted of exclusive accessory pathway conduction in Case I, coexisting normal and accessory conduction in Case 2, and exclusive normal pathway conduction in Cases 3 and 4.

Although different types of atrial tachyarrhythmias were recorded in all our patients the maximal attainable ventricular rates during the paroxysms appeared to have been related to the duration of the effective refractory period of the accessory pathway. For instance, very rapid rates (close to $300 \times \mathrm{min}$ ) were seen in Cases 2 and 4 in which the effective refractory period of the accessory pathway was shorter than that of the normal AV pathway.

In Case 2 (Fig. 4) it was so short that premature atrial impulses delivered at a coupling interval of I $70 \mathrm{msec}$ were able to reach the ventricles during the expected area of ventricular vulnerability (peak of antecedent $T$ wave).

In the presence of the multiple factors that lower the ventricular repetitive firing threshold, these early atrial impulses could have induced 'true' ventricular tachycardia or even ventricular fibrillation.

On the other hand, there were 2 patients (Cases I and 3 ) in whom the effective refractory period of the accessory pathway was longer than that of the normal pathway. Therefore the normal AV node refractoriness prevented the occurrence of the fast ventricular rates.

It should be stressed that the term effective refractory period was used as recommended by Drury (1937) in reference to the 'earliest moment at which an impulse can be liberated which will be conducted through muscle, for the response is judged by the arrival of the excitation wave at a distance or by the beat of the whole heart'. It contains, therefore, a conduction factor. Nevertheless, Drury (following Lewis' concepts) considered that this was the measurement having the greatest interest from the point of view of body surface recordings.

When the effective refractory period is measured by premature atrial stimulation, the conduction factor included in the refractory period is directly proportional to the distance between the stimulated site and the atrial end of the accessory pathway (Wellens, I97I ; Touboul et al., 1972).

Several authors (McHenry, Knoebel, and Fisch, 1966; Durrer, Schuilenburg, and Wellens, 1970; Timmis et al., I97I) also noted that, as in Case 3, AV conduction might occur through the accessory pathway only during a given part of the cycle. McHenry et al. (1966) attributed this phenomenon (which in their cases coexisted with AV block at the normal pathway) to a supernormal phase of conduction at the accessory pathway. Another explanation was offered by Wellens (I97I) and by Durrer et al. (1970). These authors postulated the existence of a functional dissociation in the upper part of the AV node with one of the two pathways connected by way of Mahaim fibres, to the upper ventricular septum. At a critical coupling interval the differences in the properties of the two pathways could have resulted in passage only through the pathway attached to the Mahaim fibre. However, a functional dissociation in the upper part of the accessory pathway totally bypassing the AV node and His bundle could also explain these findings. The same phenomenon was interpreted by Coumel et al. (1972) as being due to the combination of atrio-Hisian and His ventricular accessory pathways (connected in series or in parallel) with supernormal conduction through the latter communication. 


\section{References}

Castellanos, A., Jr., and Castillo, C. A. (1972). His bundle recordings in right bundle-branch block coexisting with iatrogenic right ventricular pre-excitation. British Heart fournal, 34, 153 .

Castellanos, A., Jr., Chapunoff, E., Castillo, C. A., Maytin, O., and Lemberg, L. (1970). His bundle electrograms in two cases of Wolff-Parkinson-White (pre-excitation) syndrome. Circulation, 4I, 399.

Castillo, C. A., and Castellanos, A., Jr. (1970). His bundle recordings in patients with reciprocating tachycardias and Wolff-Parkinson-White syndrome. Circulation, 42, 27 I.

Coumel, P., Waynberger, M., Fabiato, A., Slama, R., Aigueperse, J., and Bouvrain, Y. (1972). Wolff-Parkinson-White syndrome. Problems in evaluation of multiple accessory pathways and surgical therapy. Circulation, 45, 1216.

Coumel, P., Waynberger, M., Slama, R., and Bouvrain, Y. (197I). Interet de l'enregistrement des potentials hisiens au cors du syndrome de Wolff-Parkinson-White. Acta Cardiologica, 26, 188.

Damato, A. N., Lau, S. H., Patton, R. D., Steiner, C., and Berkowitz, W. D. (1969). A study of atrioventricular conduction in man using premature atrial stimulation and His bundle recordings. Circulation, 40, 61 .

Drury, A. N. (1937). Effective refractory period, full recovery time, and premature response interval of ventricular muscle in the intact unanaesthetised cat and rabbit. Quarterly fournal of Experimental Physiology, 26, 181.

Durrer, D., Schuilenburg, R. M., and Wellens, H. J. J. (1970). Pre-excitation revisited. American fournal of Cardiology, 25, 690 .
Massumi, R. A., and Vera, Z. (197I). Patterns and mechanisms of QRS normalization in patients with Wolff-Parkinson-White syndrome. American fournal of Cardiology, 28, $54 \mathrm{I}$.

McHenry, P. L., Knoebel, S. B., and Fisch, C. (1966). The Wolf-Parkinson-White syndrome with supernormal conduction through the accessory bypass. Circulation, 34, 734.

Moe, G. K., Mendez, C., and Han, J. (1965). Aberrant A-V impulse propagation in the dog heart: a study of functional bundle branch block. Circulation Research, 16, 261.

Timmis, G. C., Henke, J., Gordin, S., and Ramos, R. G. (197I). The Wolff-Parkinson-White syndrome in advanced atrioventricular block. American fournal of Cardiology, 28, 592.

Touboul, P., Tessier, Y., Magriña, J., Clément, C., and Delahaye, J. P. (1972). His bundle recordings and electrical stimulation of the atria in patients with Wolff-ParkinsonWhite syndrome type A. British Heart fournal, 34, 623.

Wellens, H. J. J. (1971). Electrical Stimulation of the Heart in the Study and Treatment of Tachycardias, pp. 23-26. University Park Press, Baltimore.

Wit, A. L., Damato, A. N., Weiss, M. B., and Steiner, C. (1970a). Phenomenon of the gap in atrioventricular conduction in the human heart. Circulation Research, 27, 679.

Wit, A. L., Weiss, M. B., Berkowitz, W. D., Rosen, K. M., Steiner, C., and Damato, A. N. (1970b). Patterns of atrioventricular conduction in the human heart. Circulation Research, 27, 345 .

Requests for reprints to Dr. R. J. Myerburg, Veterans Administration Hospital, Miami, Florida, U.S.A. 